2003

\title{
Controlled transformation of paramagnetism to room-temperature ferromagnetism in cobalt-doped titanium dioxide
}

\author{
A. Manivannan \\ G. Glaspell \\ M.S. Seehra
}

Follow this and additional works at: https://researchrepository.wvu.edu/faculty_publications

\section{Digital Commons Citation}

Manivannan, A.; Glaspell, G.; and Seehra, M. S., "Controlled transformation of paramagnetism to room-temperature ferromagnetism in cobalt-doped titanium dioxide" (2003). Faculty Scholarship. 100.

https://researchrepository.wvu.edu/faculty_publications/100 


\title{
Controlled transformation of paramagnetism to room-temperature ferromagnetism in cobalt-doped titanium dioxide
}

\author{
A. Manivannan, ${ }^{\text {a) }}$ G. Glaspell, and M. S. Seehra \\ Department of Physics, West Virginia University, Morgantown, West Virginia 26506
}

(Received 25 June 2003; accepted 9 September 2003)

\begin{abstract}
Samples of $\mathrm{Co}_{0.1} \mathrm{Ti}_{0.9} \mathrm{O}_{2-\delta}$ (anatase) prepared by the sol-gel technique are found to be paramagnetic at room temperature, with the magnetic susceptibility following Curie-Weiss law in the investigated range of 2-370 K. However, transformation from paramagnetism to room-temperature ferromagnetism (RTFM) is observed by hydrogenation of the sample at $573 \mathrm{~K}$. The increase in the hydrogenation time from 1 to $6 \mathrm{~h}$ increases the remanance, and the Curie temperature $\simeq 470 \mathrm{~K}$ is determined by extrapolation. X-ray photoelectron spectroscopy and transmission electron microscopy of the hydrogenated samples failed to detect Co nanoparticles, suggesting that the observed RTFM in the hydrogenated samples may be intrinsic. (c) 2003 American Institute of Physics. [DOI: 10.1063/1.1622991]
\end{abstract}

The recent reports ${ }^{1-4}$ of room-temperature ferromagnetism (RTFM) in transparent semiconducting $\mathrm{Co}_{x} \mathrm{Ti}_{1-x} \mathrm{O}_{2-\delta}$ thin films for $x \geqslant 0.01$, for both anatase and rutile phases, have attracted a great deal of attention in part because of the application of such magnetic semiconductors in spintronics. However the cause of this RTFM remains controversial since more recent reports ${ }^{5-8}$ have indicated the presence of Co nanoparticles in the thin films prepared by sputtering and pulsed laser depositions. This raises the serious possibility that the observed RTFM could be due to undetected Co nanoparticles, which because of the high Curie temperature of bulk $\mathrm{Co}\left(T_{c} \simeq 1388 \mathrm{~K}\right)$ could easily account for the observations. In a recent report from our laboratory, ${ }^{9}$ it was observed that $\mathrm{Co}_{0.1} \mathrm{Ti}_{0.9} \mathrm{O}_{2-\delta}$ anatase films prepared by a different technique, for example, spray pyrolysis are paramagnetic at room temperature. The temperature dependence of the magnetic susceptibility $\chi$ of this sample followed Curie-Weiss behavior, $\chi=\chi_{0}+C /(T-\theta)$, with $\theta \simeq-5 \mathrm{~K}$ and only for $T<5 \mathrm{~K}$ was a hysteresis loop observed. In the work presented here, we report that $\mathrm{Co}_{0.1} \mathrm{Ti}_{0.9} \mathrm{O}_{2-\delta}$ (anatase) powder prepared by the sol-gel technique is also paramagnetic at room temperature and follows a similar Curie-Weiss law. However, by controlled hydrogenation of this sample at $573 \mathrm{~K}$, the paramagnetic $\mathrm{Co}_{0.1} \mathrm{Ti}_{0.9} \mathrm{O}_{2-\delta}$ is transformed into a ferromagnet with Curie temperature $T_{c} \simeq 470 \mathrm{~K}$, without any observable change in the crystal structure as revealed by $\mathrm{x}$-ray diffraction. X-ray photoelectron spectroscopy (XPS) and transmission electron microscopy (TEM) studies of the hydrogenated sample failed to detect the presence of any Co nanoparticles. Details follow.

The sample of $\mathrm{Co}_{0.1} \mathrm{Ti}_{0.9} \mathrm{O}_{2-\delta}$ was prepared by mixing (reacting) appropriate amounts of titanium isopropoxide (Aldrich) and cobalt nitrate (Aldrich) in ethanol. The solution was dried in an oven and the powder obtained was heated to

a) Author to whom correspondence should be addressed; electronic mail: amanivan@wvu.edu
$750 \mathrm{~K}$ for $1 \mathrm{~h}$ which yielded a dark-green sample. X-ray diffraction (XRD) of this powder confirmed the anatase form of $\mathrm{TiO}_{2}$, with only a trace amount of rutile phase (Fig. 1). Part of this powder was used for magnetic measurements and the remainder used for 1,3 and $6 \mathrm{~h}$ of hydrogenation carried out at $573 \mathrm{~K}$. The hydrogen reduction setup consisted of a tubular furnace kept inside a continuously vented hood. The sample, contained in an open glass boat, was placed inside the tubular furnace and $\mathrm{H}_{2}$ gas was passed over the sample at $573 \mathrm{~K}$ with the help of a gas flow control unit. Temperature and magnetic field variations of the magnetization, $M$, of these four samples were then measured using a commercial superconducting quantum interference device (SQUID) magnetometer. These results are presented below.

The temperature variation of $\chi$ for the as-prepared samples (prior to hydrogenation) is shown in Fig. 2 where the solid line is fit to Curie-Weiss law: $\chi=\chi_{0}+C /(T-\theta)$. This variation is similar to the one reported for the sample prepared by spray pyrolysis. ${ }^{9}$ Note that the temperatures in Fig. 2 are plotted on a $\log$ scale in order to show details of the $\chi$ vs $T$ behavior at lower temperatures. From the Curie constant $C=2.6 \times 10^{-3} \mathrm{emu} \mathrm{K} / \mathrm{g}$ Oe and $C=N \mu^{2} / 3 k_{B}$ (with $N$ the number of magnetic ions/g, $k_{B}$ the Boltzmann constant and $\mu$ the magnetic moment), $\mu=4.1 \mu_{B}$ for the $\mathrm{Co}^{2+}$ ion is obtained. This magnitude of $\mu$ is consistent with the high spin state of $\mathrm{Co}^{2+}$, assuming that $\mathrm{Co}^{2+}$ substitutes for the $\mathrm{Ti}^{4+}$ ions in the anatase unit cell for $10 \%$ Co doping, with the balance of the charge compensated by $\mathrm{O}_{2-\delta}$. In the inset of Fig. 2, the linear variation of $M$ vs $H$ at $T=300 \mathrm{~K}$ is shown, confirming paramagnetism.

After hydrogenation at $573 \mathrm{~K}$, the sample acquires RTFM. The hysteresis loops were measured for samples hydrogenated for 1,3 and $6 \mathrm{~h}$, taking a new sample for hydrogenation each time. For the $6 \mathrm{~h}$ sample, Fig. 3 shows the temperature variation of the remanance $M_{r}(M$ at $H=0)$ from 5 to $350 \mathrm{~K}$ for the 1,3 and $6 \mathrm{~h}$ samples and in the inset the hysteresis loop measured at $350 \mathrm{~K}$ for the $6 \mathrm{~h}$ sample is shown. (Measurements for $T>350 \mathrm{~K}$ could not be made because of experimental limitations). These variations of $M_{r}$ vs 


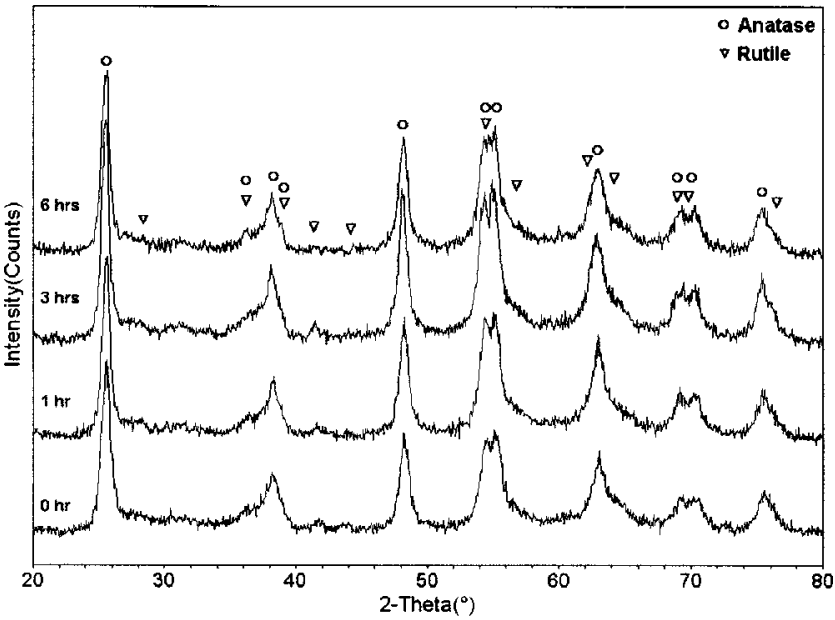

FIG. 1. X-ray diffraction patterns of the as-prepared $(0 \mathrm{~h})$ sample of $\mathrm{Co}_{x} \mathrm{Ti}_{1-x} \mathrm{O}_{2-\delta}$ and the sample hydrogenated for 1,3 and $6 \mathrm{~h}$ at $573 \mathrm{~K}$. The expected line positions for the anatase and rutile phases are shown.

$T$ are typical of a ferromagnet, with $T_{c} \simeq 470 \mathrm{~K}$ determined from extrapolating the $M_{r}$ data to zero (Fig. 3) at higher temperatures. The primary effect of increasing the hydrogenation time from 1 to $6 \mathrm{~h}$ is to increase $M_{r}$, demonstrating a similar source of the RTFM for the three samples. This is further confirmed by the temperature variation of the coercivity which also tends toward zero at $T_{c} \simeq 470 \mathrm{~K}$ (Fig. 4). Thus it appears that with the increase in hydrogenation time, more of the sample is transformed into a ferromagnet. Again, it is noteworthy that this hydrogenation does not appear to affect the crystal structure since no significant differences are observed in the XRD patterns of the four samples (Fig. 1). From the widths of the XRD lines, the average particle size of the anatase phase is $\simeq 10(2) \mathrm{nm}$, without any major changes upon hydrogenation. Although in the extrapolation of Figs. 3 and 4, similar $T_{c}$ is implified for the 1, 3 and $6 \mathrm{~h}$ samples, the extrapolations are clearly very approximate since $M_{r}$ and $H_{c}$ are often nonlinear on approach to $T_{c}$.

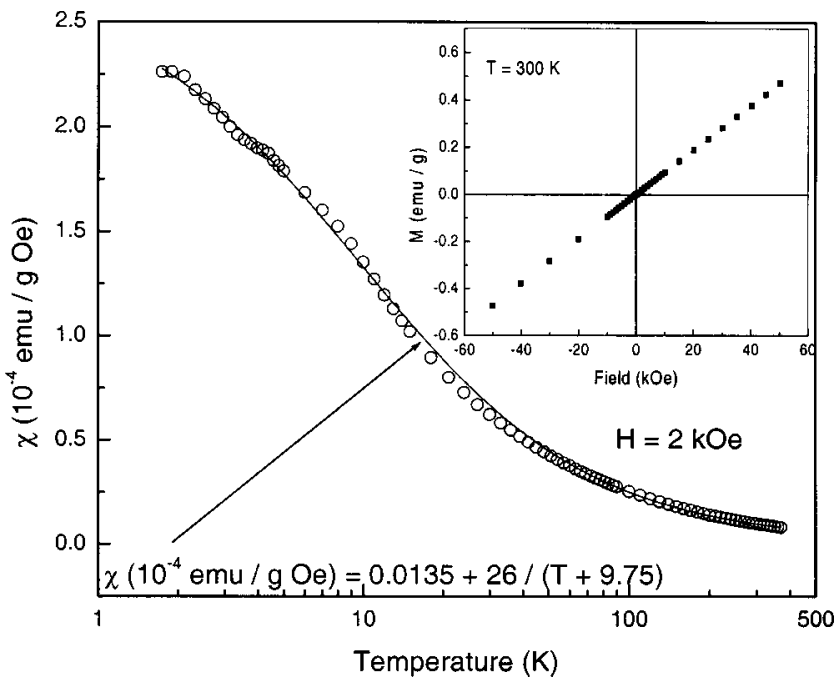

FIG. 2. Temperature dependence of the magnetic susceptibility of the asprepared sample of $\mathrm{Co}_{x} \mathrm{Ti}_{1-x} \mathrm{O}_{2-\delta}$. The solid line is fit to the equation shown. In the inset is the $M$ vs $H$ variation at $300 \mathrm{~K}$.

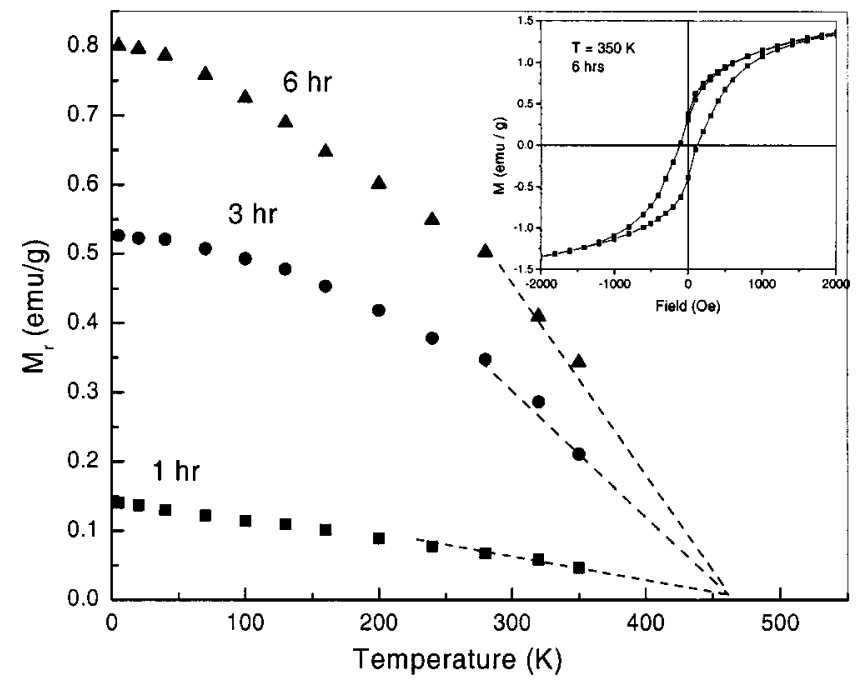

FIG. 3. Temperature variation of remanance $M_{r}$ of the sample hydrogenated for 1,3 and $6 \mathrm{~h}$ at $573 \mathrm{~K}$. The dotted lines are extrapolations, indicating $T_{c} \simeq 470 \mathrm{~K}$ where $M_{r}$ goes toward zero. In the inset, the hysteresis loop for the $6 \mathrm{~h}$ sample at $350 \mathrm{~K}$ is shown.

Thus an increase in $T_{c}$ with an increase in hydrogenation time is not ruled out for the data in Figs. 3 and 4.

In light of the recent reports on the presence of Co nanoparticles in $\mathrm{Co} / \mathrm{TiO}_{2}$ films prepared by sputtering in different partial pressures of oxygen, ${ }^{5,8}$ we carried out several experiments in addition to XRD to check for Co nanoparticles in our samples. XPS analysis of the as-prepared and the $6 \mathrm{~h}$ hydrogenated samples indicated that neither Ti nor Co is in a metallic state. For the hydrogenated sample, Ti $2 p$ peaks were observed at 457.5 and $463.2 \mathrm{eV}$ and $\mathrm{Co} 2 p$ peaks at 779.5 and $795.2 \mathrm{eV}$. The corresponding values for the asprepared samples were 458.1 and $463.8 \mathrm{eV}$ for Ti $2 p$ and 780.5 and $796.2 \mathrm{eV}$ for Co $2 p$. These magnitudes indicate $2+$ and/or $3+$ states of $\mathrm{Co}$ and $2+$ and/or $4+$ states for Ti (the metallic Co $2 p$ peak position should be at $778.3 \mathrm{eV}$ ). High resolution TEM studies of the hydrogenated samples clearly showed particles of about $10 \mathrm{~nm}$ whose energy dispersive $\mathrm{x}$-ray (EDX) spectra yielded the presence of Co, Ti

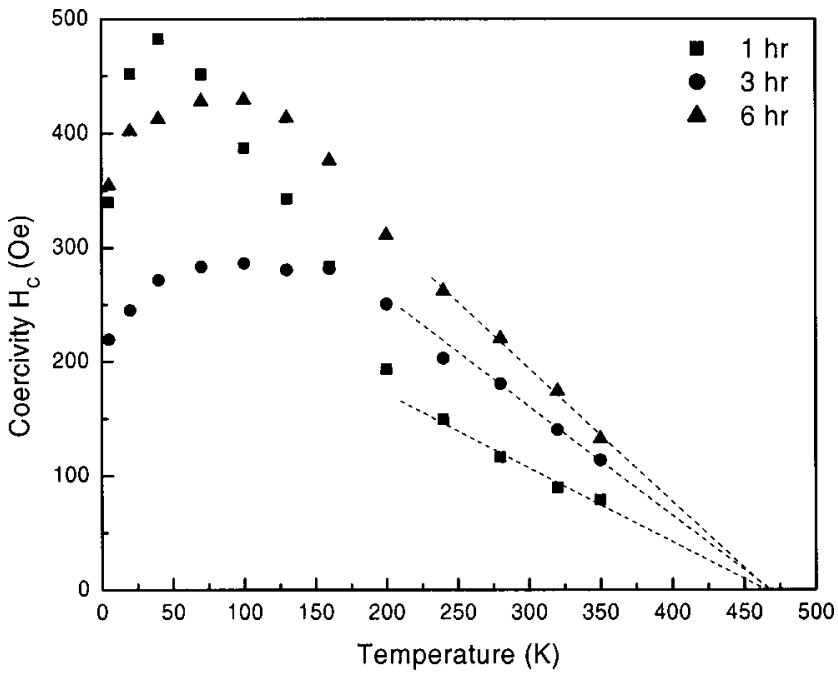

FIG. 4. Same as in Fig. 3 except that the plot is for coercivity $H_{c}$. 


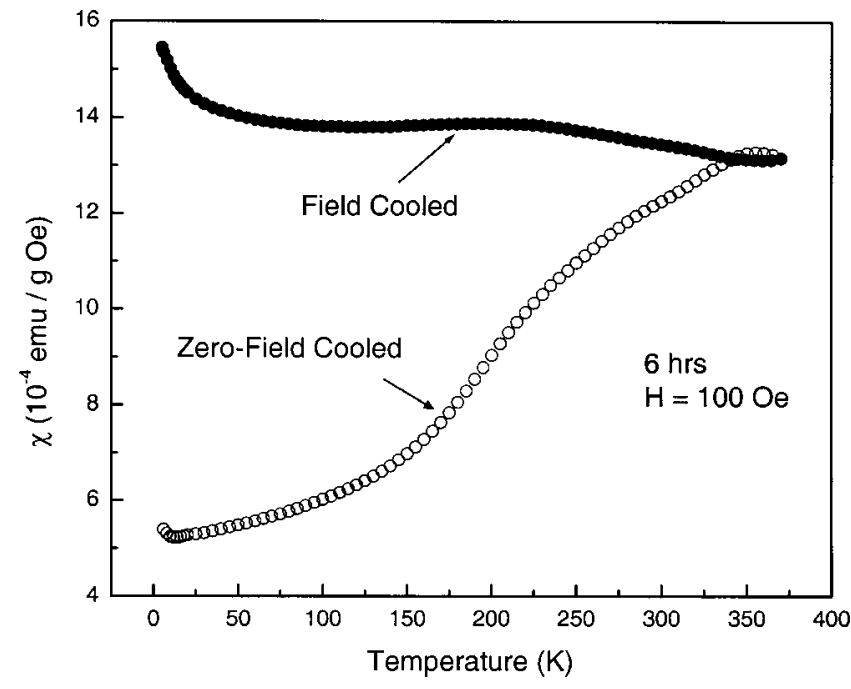

FIG. 5. Temperature variation of the magnetic susceptibility of the $6 \mathrm{~h}$ sample under zero-field-cooled and field-cooled conditions.

and oxygen, again ruling out the presence of metallic Co. Also, no exchange bias could be detected at $5 \mathrm{~K}$ in the $6 \mathrm{~h}$ sample when cooled from 300 to $5 \mathrm{~K}$ in $H=20 \mathrm{kOe}$, indicating the absence of a ferromagnetic/antiferromagnetic interface (e.g., $\mathrm{Co} / \mathrm{CoO}$ ) in the system. ${ }^{10}$ Finally, we measured $\chi$ vs $T$ for the $6 \mathrm{~h}$ sample under zero-field-cooled (ZFC) and field-cooled (FC) conditions (Fig. 5). The absence of a peak in $\chi$ for the ZFC cases, which is a signature for the blocking temperature, $T_{B}$, for Co as reported recently for thin films of $\mathrm{Co} / \mathrm{TiO}_{2}$ prepared by sputtering, ${ }^{6}$ provides additional assurance of the absence of Co nanoparticles in our samples. The reported $T_{B}$ values of Co nanoparticles of different sizes are $T_{B} \simeq 20 \mathrm{~K}(3 \mathrm{~nm}), T_{B} \simeq 50 \mathrm{~K}(6 \mathrm{~nm}), T_{B} \simeq 100 \mathrm{~K}(8 \mathrm{~nm})$ and $T_{B} \simeq 260 \mathrm{~K}(11 \mathrm{~nm}) .{ }^{11,12}$

Theoretically, it has been suggested that oxygen holes near $\mathrm{Co}$ in $\mathrm{Co}_{x} \mathrm{Ti}_{1-x} \mathrm{O}_{2-\delta}$ are essential to provide the ex- change coupling between Co ions, and lead to intrinsic RTFM. ${ }^{13}$ This indeed was the motivation for our hydrogenation experiments, since $\mathrm{H}_{2}$ is likely to extract oxygen from the sample and produce oxygen holes. From our experiments described above, we were unable to detect the presence of Co nanoparticles, thus suggesting the intrinsic nature of RTFM in our hydrogenated samples. Additional experiments such as accurate measurements of electrical conductivity with time of hydrogenation would be useful to confirm these observations.

The authors express their thanks to C. Stinespring for providing facilities for the hydrogenation experiments and $\mathrm{J}$. Poston of National Energy Technology Laboratory, Morgantown, for the XPS experiments. TEM measurements were done by Advanced Materials Engineering Inc. Sunnyvale, CA. This research was supported in part by the U.S. Air Force Office of Scientific Research.

${ }^{1}$ Y. Matsumoto et al., Science 291, 854 (2001).

${ }^{2}$ S. A. Chambers et al., Appl. Phys. Lett. 79, 3467 (2001).

${ }^{3}$ Y. Matsumoto et al., Jpn. J. Appl. Phys., Part 2 40, L1205 (2001).

${ }^{4}$ W. K. Park, R. J. Ortega-Hertogs, J. Moodera, A. Punnoose, and M. S. Seehra, J. Appl. Phys. 91, 8093 (2002)

${ }^{5}$ P. A. Stampe, R. J. Kennedy, Y. Xin, and J. S. Parker, J. Appl. Phys. 93, 7864 (2003).

${ }^{6}$ A. Punnoose, M. S. Seehra, W. K. Park, and J. S. Moodera, J. Appl. Phys. 93, 7867 (2003).

${ }^{7}$ D. H. Kim et al., J. Appl. Phys. 93, 6125 (2003)

${ }^{8}$ B. Z. Rameev, F. Yildiz, L. R. Tagirov, B. Aktas, W. K. Park, and J. S. Moodera, J. Magn. Magn. Mater. 258-259, 361 (2003).

${ }^{9}$ A. Manivannan, M. S. Seehra, S. B. Majumder, and R. S. Katiyar, Appl. Phys. Lett. 83, 111 (2003).

${ }^{10}$ A. E. Berkowitz and K. Takano, J. Magn. Magn. Mater. 200, 552 (1999), and references therein.

${ }^{11}$ J. P. Chen, C. M. Sorensen, K. J. Klabunde, and G. C. Hadjipanayis, Phys. Rev. B 51, 11527 (1995).

${ }^{12}$ C. B. Murray, S. Sun, H. Doyle, and T. Betley, MRS Bull. 26, 985 (2001).

${ }^{13}$ M. S. Park, S. K. Kwon, and B. I. Min, Phys. Rev. B 65, 161201 (2002). 